\title{
Frictional dependence of shallow-granular flows from discrete particle simulations
}

\author{
Anthony R. Thornton ${ }^{1,2, a}$, Thomas Weinhart ${ }^{1,2}$, Stefan Luding ${ }^{1}$, and Onno Bokhove ${ }^{2,3}$ \\ 1 Multi-Scale Mechanics, Department of Mechanical Engineering, University of Twente, P.O. Box 217, 7500 AE Enschede, The \\ Netherlands \\ 2 Mathematics of Computational Science, Department of Applied Mathematics, University of Twente, P.O. Box 217, 7500 AE \\ Enschede, The Netherlands \\ 3 School of Mathematics, University of Leeds, Leeds, LS2 9JT, UK
}

Received 28 April 2012 and Received in final form 3 September 2012

Published online: 7 December 2012

(c) The Author(s) 2012. This article is published with open access at Springerlink.com

\begin{abstract}
A shallow-layer model for granular flows down inclines is completed with a closure relation for the macroscopic bed friction obtained from micro-scale, discrete particle simulations of steady flows over geometrically rough bases with contact friction. Microscopic friction can be different between bulk particles and with particles at the base, where the latter is systematically varied. When extending the known friction closure relation to be a function of both bulk flow and bed properties, surprisingly, we find that the macroscopic bed friction is only weakly dependent on the contact friction of the bed particles and is predominantly determined by the properties of the flowing particles. Implications for constitutive modelling and possible experiments to better understand the bulk rheology are discussed.
\end{abstract}

\section{Introduction}

Free-surface flows of granular material occur in many geophysical situations and engineering applications, such as rockslides, avalanches, or production-line transport. They have been studied extensively both experimentally and numerically. The most direct way to simulate granular flows is by methods such as the Discrete Particle Method (DPM), which computes the movement of individual particles based on a model of the contact forces between the particles $[1,2]$. However, realistic flow situations often involve billions of particles, and can only be modelled on a coarser level by continuum solvers (or hybrid methods), in which the particulate flow is described by a small number of continuum fields governed by the conservation of mass, momentum, and often energy. For shallow flows, the mass and momentum conservation equations can be further simplified by averaging over the flow depth, yielding granular shallow-layer equations (GSLE) [3-5]. In order to obtain a closed system of equations, closure relations for the normal stress differences, velocity shape factor, and macro basal friction have to be provided in terms of the flow variables: height (more correctly this should be called flow thickness; however, here we follow the convention in the literature and call this variable height), $h$, and the

\footnotetext{
a e-mail: a.r.thornton@utwente.nl
}

depth-averaged velocity, $\overline{\boldsymbol{u}}=(\bar{u}, \bar{v})$. While closure models are usually developed to retain the qualitative behaviour of the microscopic system, they often cannot describe the quantitative behaviour as the relations between the microand macroscopic quantities are not well known. Here, we focus on one closure relation: the effective macro-friction coefficient $\mu=\mu(h,|\overline{\boldsymbol{u}}|)$ and its dependence on the bed friction.

Concerning nomenclature: in the literature, the word friction is used for both the macroscopic frictional forces felt by a large mass of material moving over a surface, and the contact frictional force between two individual particles, i.e., the contact friction used in the DPM simulation. Here, the macroscopic (shallow-layer) friction will be denoted as $\mu$, the particle-particle contact friction between flowing particles as $\mu^{f}$, and the contact friction between flowing and base particles as $\mu^{b}$.

The effective macro-friction coefficient, $\mu$, determines the range of inclinations and heights at which the flow either arrests, reaches steady flow, or accelerates indefinitely. The rougher the base, the larger the range of inclinations at which steady flow is reached. Basal roughness can be realised in various ways. Goujon et al. [6] created a rough base by glueing particles onto a flat surface. The roughness was changed by varying the diameter ratio between fixed basal and free-flowing particles. They observed a peak in the measured macro-friction coefficient at a cer- 
tain diameter ratio depending on the compactness of the basal layer. In their work on enduring contacts, Louge and Keast [7] modelled the base as a flat frictional incline. Later, Louge [8] extended their theory to bumpy, geometric rough, inclines. Silbert et al. $[9,10]$ used DPM to simulate chute flow over a base of disordered particles and obtained the closure relations as a function of flow height and velocity ${ }^{1}$.

In our research we aim to obtain the closure relations as a function of the basal properties, as well as bulk properties, by studying relatively small steady-state DPM simulations. The ultimate aim is to be able to perform continuum GSLE simulations in complex geometries with spatially and temporally evolving basal properties. First, we developed a statistical method in [12] to extract the continuum fields from the microscopic degrees of freedom that is also valid near the base of the flow. Then, an extensive parameter study was undertaken in [13] to obtain the full set of closure laws for the GSLE. Here, we study the closure relation for the macro-friction coefficient as a function of the contact friction between basal and flowing particles, $\mu^{b}$.

\section{Mathematical background}

\subsection{Granular shallow-layer model}

GSLE have proved to be a successful tool in predicting both geological large-scale [14-18] and laboratory-scale experiments $[4,19-21]$ of granular chute flows. They have been derived in many papers, starting with [5], but here we use the form presented in $[3,4]$.

We will consider the flow down a slope with inclination $\theta$, the $x$-axis downslope, $y$-axis across the slope and the $z$-axis normal to the slope. In general, the freesurface and base locations are given by $z=s(x, y)$ and $z=b(x, y)$, respectively. Here, we will only consider flows over rough flat surfaces where $b$ can be taken as constant. The height of the flow is $h=s-b$ and the velocity components are $\boldsymbol{u}=(u, v, w)^{T}$. Depth-averaging the mass and momentum balance equations and retaining only leading and first-order terms (in the ratio of height to length of the flow) yields the depth-averaged shallow-granular equations, e.g., [4],

$$
\begin{gathered}
\frac{\partial h}{\partial t}+\frac{\partial}{\partial x}(h \bar{u})+\frac{\partial}{\partial y}(h \bar{v})=0 \\
\frac{\partial}{\partial t}(h \bar{u})+\frac{\partial}{\partial x}\left(h \bar{u}^{2}+\frac{g}{2} h^{2} \cos \theta\right)+\frac{\partial}{\partial y}(h \bar{u} \bar{v})=S_{x} \\
\frac{\partial}{\partial t}(h \bar{v})+\frac{\partial}{\partial x}(h \bar{u} \bar{v})+\frac{\partial}{\partial y}\left(h \bar{v}^{2}+\frac{g}{2} h^{2} \cos \theta\right)=S_{y}
\end{gathered}
$$

where $g$ is the gravitational acceleration, $\overline{\boldsymbol{u}}=(\bar{u}, \bar{v})$ the depth-averaged velocity and the source terms are given by

$$
S_{x}=g h \cos \theta\left(\tan \theta-\mu \frac{\bar{u}}{\sqrt{\bar{u}^{2}+\bar{v}^{2}}}\right)
$$

\footnotetext{
${ }^{1}$ After initial submission we became aware of [11] which
} looks at layering effects in the same flow configurations. and

$$
S_{y}=g h \cos \theta\left(-\mu \frac{\bar{v}}{\sqrt{\bar{u}^{2}+\bar{v}^{2}}}\right) .
$$

This granular shallow-layer theory assumes that the flow is incompressible, the stress is isotropic and the velocity profile is uniform in depth. When these assumptions are accepted the only remaining closure relation is $\mu$. We note that some of these assumptions can be relaxed by introducing closure relations for the mean density, the normal stress differences, and the shape of the velocity profile. This, however, is beyond the scope of this paper; we refer the interested reader to [13].

\subsection{Friction law for rough surfaces}

The closure to eqs. (1) is achieved by determining the bed macro-friction in terms of the flow variables, such that $\mu=\mu(h,|\overline{\boldsymbol{u}}|)$. In the early models a constant friction coefficient was used $[5,22]$, i.e., $\mu=\tan \delta$, where $\delta$ is a fixed basal frictional angle. For these models, steady uniform flow is only possible at a single inclination, $\delta$, below which the flow arrests, and above which the flow accelerates indefinitely. However, detailed experimental investigations [23-25] for the flow over rough uniform beds showed that steady flow emerges at a range of inclinations, $\theta_{1}<\theta<\theta_{2}$, where $\theta_{1}$ is the minimum angle required for flow, and $\theta_{2}$ is the maximum angle at which steady uniform flow is possible. In [25], the measured height $h_{\text {stop }}(\theta)$ of stationary material left behind when a flowing layer has been brought to rest, was fitted to

$$
\frac{h_{\text {stop }}(\theta)}{A d}=\frac{\tan \left(\theta_{2}\right)-\tan (\theta)}{\tan (\theta)-\tan \left(\theta_{1}\right)}, \quad \theta_{1}<\theta<\theta_{2},
$$

where $d$ is the particle diameter and $A$ a characteristic dimensionless scale over which the friction varies. Here, we will investigate how the parameters $A, \theta_{1}$ and $\theta_{2}$ change as a function of the contact friction between bed and flowing particles.

For $h>h_{\text {stop }}$, steady flow exists where the Froude number, $F=|\overline{\boldsymbol{u}}| / \sqrt{g h \cos \theta}$, is assumed to fit a linear function of height

$$
F=\frac{\beta h}{h_{\text {stop }}(\theta)}-\gamma, \quad \theta_{1}<\theta<\theta_{2},
$$

where $\beta$ and $\gamma$ are constants independent of the chute inclination and particle size.

From eqs. (2) and (3) we can derive a relation between the inclination $\theta$ and the flow variables $F$ and $h$. For steady flow over a uniform bed, the momentum eqs. (1) reduce to $\mu=\tan \theta$, and by combining this with (2) and (3), we obtain the friction law

$$
\mu(h, F)=\tan \left(\theta_{1}\right)+\frac{\tan \left(\theta_{2}\right)-\tan \left(\theta_{1}\right)}{\beta h /(A d(F+\gamma))+1} .
$$

Even though (4) is derived for steady-flow conditions, it is expected to hold, in an asymptotic sense, for unsteady situations; therefore, we can attempt to use it as the closure relation for (1). 


\section{Problem description}

\subsection{Contact description}

We perform simulations of a collection of mono-dispersed spherical granular particles of diameter $d$ and density $\rho_{p}$; each particle $i$ has a position $\boldsymbol{r}_{i}$, velocity $\boldsymbol{v}_{i}$ and angular velocity $\boldsymbol{\omega}_{i}$. We assume that particles are soft and each contact area is relatively small and, hence, can be treated as point-like. The relative distance is $r_{i j}=\left|\boldsymbol{r}_{i}-\boldsymbol{r}_{j}\right|$, the unit normal $\hat{\boldsymbol{n}}_{i j}=\left(\boldsymbol{r}_{i}-\boldsymbol{r}_{j}\right) / r_{i j}$ and the relative velocity $\boldsymbol{v}_{i j}=\boldsymbol{v}_{i}-\boldsymbol{v}_{j}$. Two particles are in contact if their overlap, $\delta_{i j}^{n}=\max \left(0, d-r_{i j}\right)$, is positive. The normal and tangential relative velocities at the contact point are given by

$$
\begin{aligned}
\boldsymbol{v}_{i j}^{n} & =\left(\boldsymbol{v}_{i j} \cdot \hat{\boldsymbol{n}}_{i j}\right) \hat{\boldsymbol{n}}_{i j}, \\
\boldsymbol{v}_{i j}^{t} & =\boldsymbol{v}_{i j}-\boldsymbol{v}_{i j}^{n}+\frac{d-\delta_{i j}^{n}}{2} \hat{\boldsymbol{n}}_{i j} \times\left(\boldsymbol{\omega}_{i}+\boldsymbol{\omega}_{j}\right) .
\end{aligned}
$$

The total force on particle $i$ is a combination of the contact forces $\boldsymbol{f}_{i j}^{n}+\boldsymbol{f}_{i j}^{t}$ between two particles $i, j$ in contact and external forces, which for this investigation will be limited to gravity, $m \boldsymbol{g}$. For interactions the normal, $\boldsymbol{f}_{i j}^{n}$, and tangential, $\boldsymbol{f}_{i j}^{t}$, forces have to be modelled. Initially we will consider three different interactions: spring-dashpot, Hertzian and plastic. For each model, when the tangentialto-normal force ratio becomes larger than a contact friction coefficient, $\mu^{c}$, the tangential force yields and the particles slide, and we truncate the magnitude of the tangential force as necessary to satisfy $\left|\boldsymbol{f}_{i j}^{t}\right| \leq \mu^{c}\left|\boldsymbol{f}_{i j}^{n}\right|$. Here $\mu^{c}=\mu^{f}$ for contacts between two flowing particles and $\mu^{b}$ for contacts between flow and basal particles. We integrate the resulting force and torque relations in time using Velocity-Verlet and forward Euler [26] with a time step $\Delta t=t_{c} / 50$, where $t_{c}$ is the collision time [2]. We distinguish between free flowing and fixed bed particles. The fixed bed particles are modelled as having an infinite mass and are unaffected by body and contact forces: they do not move. This leaves two distinct types of collision: flow-flow, and flow-base. Model parameters for each of these collision types are changed independently and are distinguished by a subscript $f$ for flow-flow, $b$ for flow-base collision parameters, respectively.

For the spring-dashpot case $[1,2,13]$ the normal, $\boldsymbol{f}_{i j(s d)}^{n}$, and tangential, $\boldsymbol{f}_{i j(s d)}^{t}$, forces are modelled with linear elastic and linear dissipative contributions, hence

$$
\boldsymbol{f}_{i j(s d)}^{n}=k^{n} \delta_{i j}^{n} \hat{\boldsymbol{n}}_{i j}-\gamma^{n} \boldsymbol{v}_{i j}^{n}, \boldsymbol{f}_{i j(s d)}^{t}=-k^{t} \boldsymbol{\delta}_{i j}^{t}-\gamma^{t} \boldsymbol{v}_{i j}^{t},
$$

with spring constants $k^{n}, k^{t}$ and damping coefficients $\gamma^{n}$ $\gamma^{t}$. The elastic tangential displacement. $\boldsymbol{\delta}_{i j}^{t}$, is defined to be zero at the initial time of contact, and its rate of change is given by

$$
\frac{\mathrm{d}}{\mathrm{d} t} \boldsymbol{\delta}_{i j}^{t}=\boldsymbol{v}_{i j}^{t}-r_{i j}^{-1}\left(\boldsymbol{\delta}_{i j}^{t} \cdot \boldsymbol{v}_{i j}\right) \boldsymbol{n}_{i j}
$$

In eq. (7), the first term is the relative tangential velocity at the contact point, and the second term ensures that $\boldsymbol{\delta}_{i j}^{t}$ remains normal to $\boldsymbol{n}_{i j}$, see [13] for details.
For the Hertzian case we modify the interaction force with

$$
\boldsymbol{f}_{i j(\text { Hertz })}^{n / t}=\sqrt{\delta_{i j}^{n} / d} \boldsymbol{f}_{i j(s d)}^{n / t},
$$

see, e.g., [9]. Finally, for the plastic case we modify the normal force using the (hysteretic) elastic-plastic form of Walton and Braun, e.g. [2,27]; therefore, in the normal direction a different spring constant is taken for loading and unloading/reloading of the contact and no dash-pot is used i.e.,

$$
\begin{gathered}
\boldsymbol{f}_{i j(p)}^{n}=\left\{\begin{array}{lll}
k_{1}^{n} \delta_{i j}^{n} \hat{\boldsymbol{n}}_{i j}, & \text { if } \quad k_{2}^{n} \delta_{i j}^{e} \geq k_{1} \delta_{i j}^{n}, \\
k_{2}^{n} \delta_{i j}^{e} \hat{\boldsymbol{n}}_{i j}, & \text { if } \quad k_{1}^{n} \delta_{i j}^{n}>k_{2}^{n} \delta_{i j}^{e}>0, \\
\mathbf{0}, & \text { if } \quad 0 \geq k_{2}^{n} \delta_{i j}^{e},
\end{array}\right. \\
\boldsymbol{f}_{i j(p)}^{t}=\boldsymbol{f}_{i j(s d)}^{t}=-k^{t} \boldsymbol{\delta}_{i j}^{t}-\gamma^{t} \boldsymbol{v}_{i j}^{t},
\end{gathered}
$$

with $\delta_{i j}^{e}=\delta_{i j}^{n}-\delta_{i j}^{\max }\left(1-k_{1}^{n} / k_{2}^{n}\right)$ and, $\delta_{i j}^{\max }=\max \delta_{i j}^{n}$ is the maximum overlap during the contact. Unlike $[2,27]$ we take $k_{2}^{n}$ to be constant, so that the normal coefficient of restitution is given by $\epsilon_{n}=\sqrt{k_{2}^{n} / k_{1}^{n}}$. However for enduring contacts the dissipation is smaller than in the springdashpot case, since oscillations on the unloading/reloading $\left(k_{2}^{n}\right)$ branch do not dissipate energy. This leads to less total dissipation, which could be responsible for the small differences observed between the plastic and the springdashpot contact model (see sect. 4.1 for more discussion). For a more detailed review of contact laws, in general, we refer the reader to [2].

In the following simulations, parameters are nondimensionalised such that the flow particle diameter $d=1$, mass $m=1$ and the magnitude of gravity $g=1$. For the springdashpot and Hertzian cases, the normal spring and damping constants are $k^{n}=2 \cdot 10^{5}$ and $\gamma^{n}=50$. The tangential spring and damping constants are $k^{t}=(2 / 7) k^{n}$ and $\gamma^{t}=\gamma^{n}$. This means that in the spring-dashpot case: the frequency of normal and tangential contact oscillation and the normal and tangential dissipation are equal; the contact duration is $t_{c}=0.005$; and, the normal coefficient of restitution is $\epsilon_{n}=0.88$. These parameters are identical to those used by Silbert et al. [9] except that a dissipation in the tangential direction, $\gamma^{t}$, was added to better dampen rotational degrees of freedom in arresting flow.

In the Hertzian case the same values as in the springdashpot case were used for $k^{n}, k^{t}, \gamma^{t}$ and $\gamma^{n}$; but, due to the nonlinearity in the Hertzian contact model the coefficient of restitution becomes velocity dependent. For a typical simulation velocity of 1.06 the coefficient of restitution, $\epsilon_{n}$, is around 0.98 .

For the plastic case the $k_{2}^{n}$ was taken to be the same as the $k^{n}$ in the spring-dashpot case and $k_{1}^{n}=k_{2}^{n} / \epsilon_{n}^{2}$ was chosen such that the normal coefficients of restitution are identical in both cases.

We performed a detailed study of the effect of changing the contact model for the case $\mu^{b}=\mu^{f}$, see fig. 1 . The effect of changing the contact model was minor with the only noticeable difference occurring for low inclinations, $\theta$, where in the Hertzian case the $h_{\text {stop }}$ curve diverges to infinity at an angle around $2^{\circ}$ higher; a more 


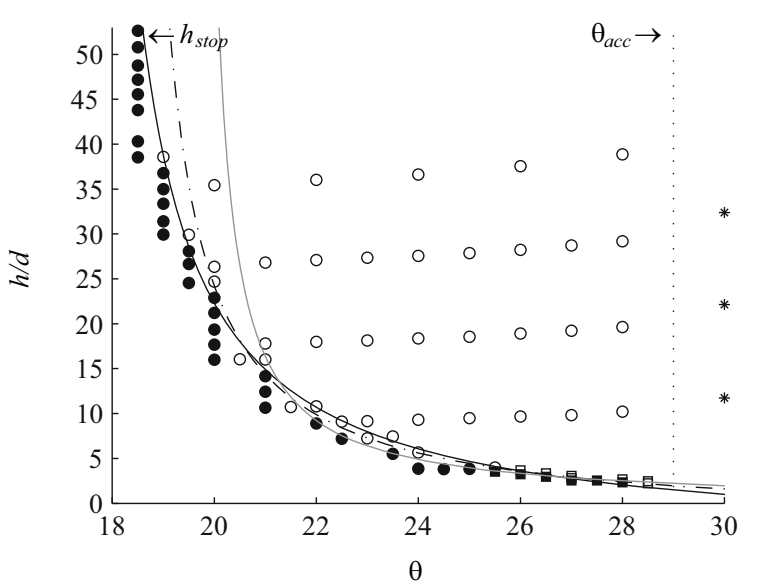

Fig. 1. Overview of DPM simulations for $\mu^{f}=\mu^{b}=0.5$, with markers denoting the different states: arrested (solid symbols), steady (open symbols), and accelerating $(*)$. The demarcation line is fitted to $h_{\text {stop }}$, see eq. (2) (solid line). Circles denote increasing number of particles and squares decreasing. The grey line shows results using the Hertzian contact model, whereas the dot-dashed line shows the results using the plastic model.

detailed discussion of the effects of the contact law can be found in sect. 4.1. In the absence of tangential forces a narrow Pouliquen style $h_{\text {stop }}$ curve is still generated; however, the angles are considerably lower than those observed in experiments (data not shown). The simple linear springdashpot model has the major advantage that we can be sure that nonlinearities and interesting phenomena we observe are not due to the contact model, but are fundamental to the flow configuration and rheology. For this reason, and the fact that changing to a more complex interaction model does not change the phenomena, we will use this contact model to undertake a detailed investigation of the effect of changing $\mu^{b}$ on the macroscopic friction, $\mu$. In this study, the friction between bed and flowing particles, $\mu^{b}$, is varied between $\mu^{b}=0$ and $\infty$; whereas, $\mu^{f}$ is fixed at 0.5 .

\subsection{Chute geometry}

The chute is periodic and of size $20 d \times 10 d$ in the $x \times y$ directions, with inclination $\theta$. The base is created by performing a 12 particle deep simulation, across a flat surface, relaxing the system and then taking a 1.7 particle deep cross-section to use as a rough bottom; for details see [13].

The height of the flow is determined by the number of bulk flowing particles, $N$, which are initially randomly distributed with a low packing fraction of about $\rho / \rho_{p}=0.3$. From this state the particles accelerate and compact to a height of approximately, $N / 200$, giving the chute enough kinetic energy to initialise flow. A screen shot of a system in steady state is given in fig. 2 .

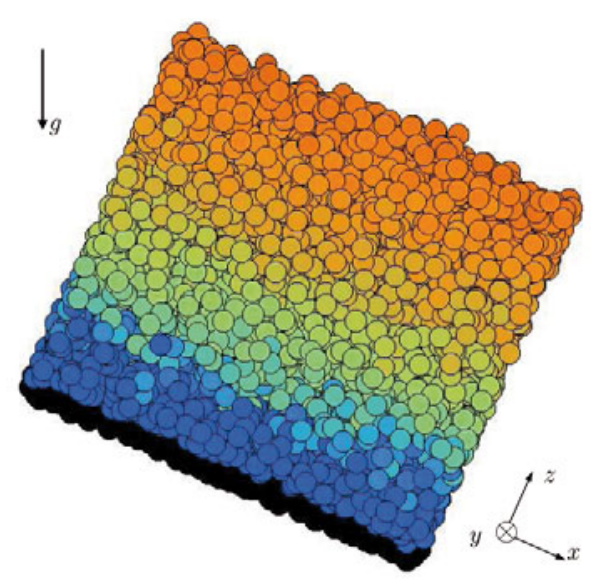

Fig. 2. DPM simulation for 3500 particles, in a chute inclination at $\theta=24^{\circ}$ with a basal contact friction, $\mu^{b}=0.5$, at time $t=2000$; gravity direction $\boldsymbol{g}$ as indicated. The domain is periodic in the $x$ - and $y$-directions. In the plane normal to the $z$-direction, fixed (black) particles form a rough base while the surface is unconstrained. Colours indicate speed, which increase from slow (blue) at the bottom to faster (orange) towards the free surface.

\subsection{Statistics}

To obtain macroscopic fields, we use the coarse-graining statistical methods as described in [28,29], extended to incorporate external boundary forces [12]. For this statistical method a coarse-graining function that spatially smears the discrete data has to be defined; we use a Gaussian of width, or variance, $d / 4$. The height of the flow is defined to be the distance between the point where the downwards normal stress $\sigma_{z z}$ vanishes and where it reaches its maximum value. In order to avoid the effects of coarse graining, the linear bulk stress profile between $2 \%$ and $98 \%$ of its maximum is linearly extrapolated to define the base and surface locations (see [13] for details).

We perform two types of simulation: $h_{\text {stop }}$ and dynamic. For the dynamic simulations the flow is assumed to be steady at $t=2000$ if the kinetic energy shows no drift over the interval $1500<t<2000$. To obtain depth profiles of the macroscopic fields in steady state, an average is taken over $t \in[2000,2100]$ and the $x$ - and $y$-directions.

The $h_{\text {stop }}$ simulations determine the demarcation line between arrested and steady flow with good accuracy (the $h_{\text {stop }}$ curve), via the following algorithm: starting with $N=1200$ flow particles and inclination $\theta=24^{\circ}$, the angle was increased in steps of $1^{\circ}$ until a flowing state was reached. If the flow arrested, the number of particles was increased by 400 or else the angle decreased by $1 / 2^{\circ}$ (circles in fig. 1). Flow was defined to be arrested when the ratio between the kinetic energy and the elastic energy stored in the contact, $E_{\text {kin }} / E_{\text {ela }}$, fell below $10^{-5}$ before $t=500$ was reached, otherwise the flow was determined as flowing. In contrast to [13], we also determined the demarcation line for thin flows (for the spring-dashpot model only): starting with $N=800$ and $\theta=21^{\circ}$, the angle was increased by $1 / 2^{\circ}$, if the flow arrested, otherwise the 
Table 1. Table showing the measured values of $\theta_{1}, \theta_{2}, A, \beta$ and $\gamma$ for the case $\mu^{b}=\mu^{f}$ for all three contact models considered.

\begin{tabular}{c|ccc||cc}
\hline & $\theta_{1}$ & $\theta_{2}$ & $A$ & $\beta$ & $\gamma$ \\
\hline $\begin{array}{c}\text { Spring-dashpot }\left({ }^{\mathrm{a}}\right) \\
\text { (without low } h)\end{array}$ & 17.7 & 32.8 & 3.36 & 0.196 & 0.40 \\
Spring-dashpot & 17.6 & 34.4 & 3.16 & 0.217 & 0.0699 \\
Plastic & 18.3 & 37.9 & 1.91 & 0.244 & 0.145 \\
Hertzian & 19.7 & 88.2 & 0.0134 & 0.321 & 0.318 \\
\hline
\end{tabular}

${ }^{(a)}$ Data taken from [13], where the flows with heights for which $N / 200 \approx h / d$ is less than 5 were not included in the $h_{\text {stop }}$ curves.

number of particles was decreased by $10 \%$ until $N<200$ was reached (squares in fig. 1).

From the experiments of Pouliquen [24], steady granular flow over a rough base is known to exist for a range of heights and inclinations, $\theta_{\text {stop }}(h)<\theta<\theta_{\text {acc }}$, where $\theta_{\text {stop }}(h)$ denotes the inverse function of $h_{\text {stop }}(\theta)$. From the $h_{\text {stop }}$ simulations we obtain inclination intervals at various heights and height intervals at various inclinations between which the actual demarcation line lies, see fig. 1 . The demarcating curve was then fitted to eq. (2) by minimising the distance of the fit to these intervals. Note that these simulations are shorter than the dynamic simulations, due to the large number of simulations required to obtain high-resolution $h_{\text {stop }}$ curves.

\section{Results}

\subsection{Effect of the contact model}

It can be seen from fig. 1 that changing the contact model does not have a large effect on the shape of the $h / h_{\text {stop }}$ curve; however, it does significantly change the fitting parameters, see table 1 . It can be seen that $\theta_{1}$ is reasonably stable, which is expected, as it is related to the angle of repose of the material [30]; whereas, $\theta_{2}$, which is an extrapolated angle, is more poorly defined. Small changes in the data can lead to large changes in the values obtained for $A$ and $\theta_{2}$. This is highlighted by the first two rows in table 1 , where fits are computed, for the spring-dashpot case, with and without the $h / d \approx N / 200 \leq 5$ data. Including this data increases $\theta_{2}$ and decreases $A$, which is a similar effect to changing the interaction model from spring-dashpot to plastic. Changing to the Hertzian contact model follows the same trend, but with a much larger effect on $\theta_{2}$ and $A$. The sensitivity of $A$ and $\theta_{2}$ to small changes in the data is discussed in more detail in sect. 4.3.

From fig. 3 it is clear that for all three contact models there is a linear relationship between the Froude number and the scaled height, $h / h_{\text {stop }}$; however, the gradient of this curve does depend on the details of the contact model. The trend is that the gradient, $\beta$, decreases in line with the total dissipation inherent in the contact model, i.e., a more dissipative contact model leads to a lower value of $\beta$.

The key results of this section are: changing the contact model does not affect the linear scaling of Froude number

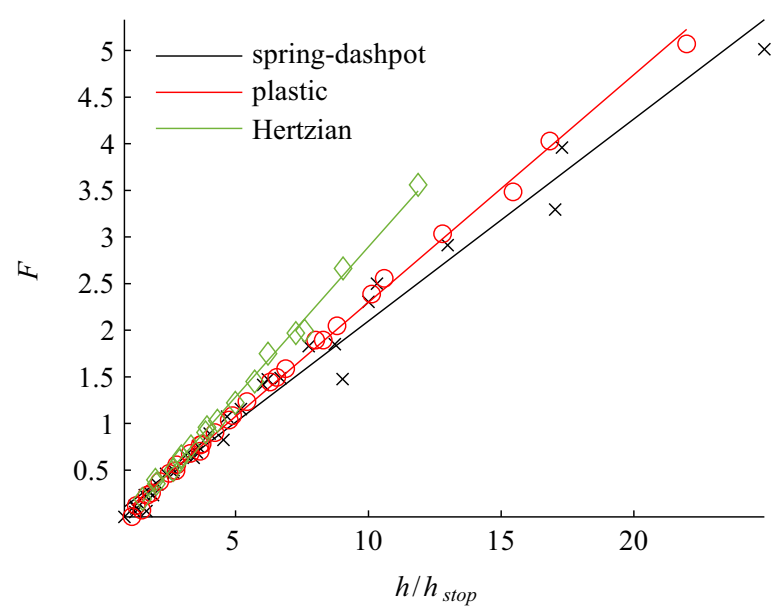

Fig. 3. Froude number $F=\bar{u} / \sqrt{g h}$ versus height scaled by the stopping height, $h_{\text {stop }}$ for $\mu^{b}=\mu^{f}$ and all three considered contact models.

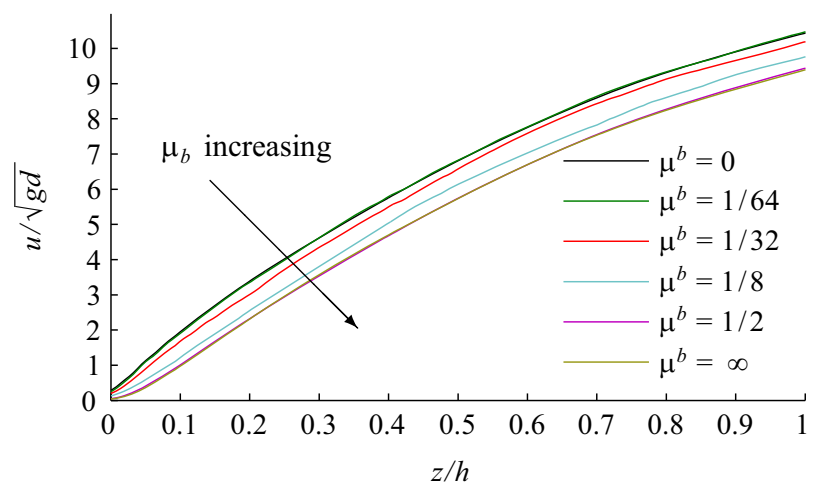

Fig. 4. Flow velocity profile for thick flow with $N=6000$ $(H=30)$, inclination $\theta=24^{\circ}$ and bed micro-friction $\mu^{b}=$ $0,1 / 1024,1 / 2, \infty$. The flow velocity roughly observes a Bagnold profile $\left(u(z)=5 / 3 \bar{u}\left(1-(1-z / h)^{3 / 2}\right)\right)$, except near the surface and the base.

against $h / h_{\text {stop }}$ and does not change the shape of the $h_{\text {stop }}$ curve; however, it does affect the details of the fits. The Pouliquen law is universal with respect to the contact law used in the DPM simulations.

\subsection{Frictional dependence in the depth profiles}

For all simulations we observe nearly constant density profiles, and linear stress profiles for $\sigma_{z z}$ and $\sigma_{x z}$, with depth. These satisfy the mass and momentum balances for steady uniform flow. Additionally, we do find a small normal stress anisotropy, i.e., $\sigma_{x x} \neq \sigma_{z z}$. Figure 4 shows a selection of velocity profiles. We observe a Bagnold profile as predicted in [31] for thick collisional flows. A small deviation from the Bagnold profile is observed at the surface, where the profile becomes linear and near the base where the shear rate decreases. For $\mu^{b}<1 / 2$, the flow shows a slip velocity at the base, a characteristic of smoother bases. In [13] it was shown that both the bulk density and the shape of the velocity profile depend on the inclination and height of the flow. 


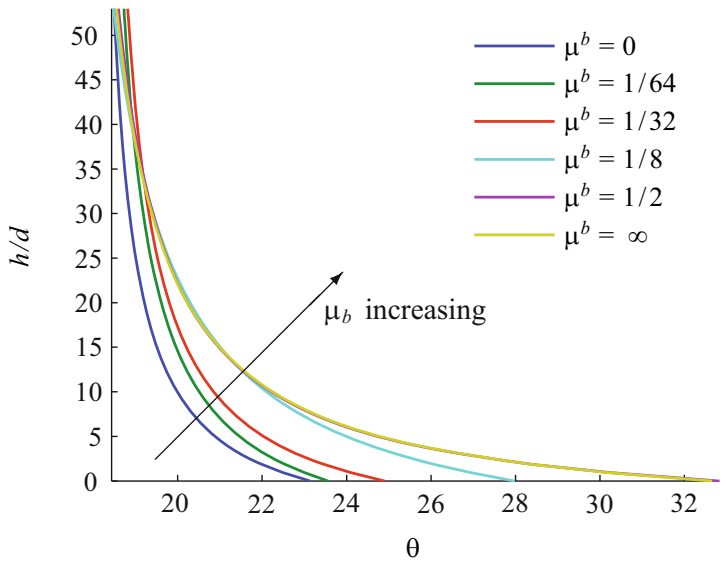

Fig. 5. Demarcation lines $h_{\text {stop }}\left(\theta ; \mu^{b}\right)$ between retarding and steady flows for various values of $\mu^{b}$. The demarcation line is fitted to eq. (2). Note that the line $\mu^{b}=1 / 2$ and $\mu^{b}=\infty$ nearly coincide.

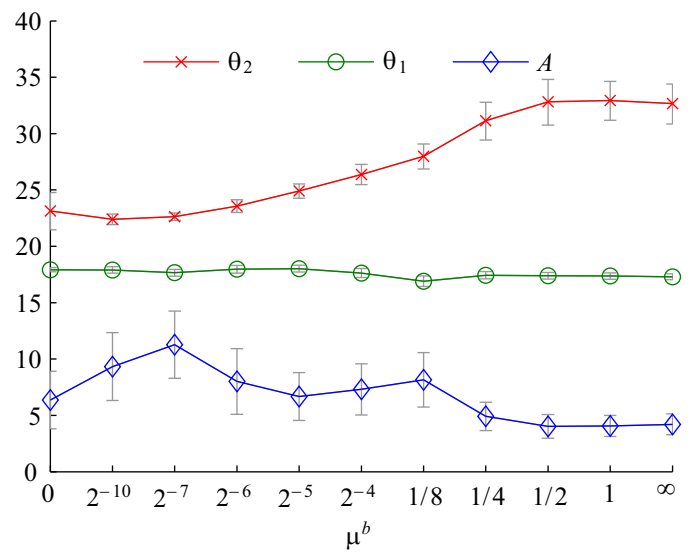

Fig. 6. Dependence of $A, \theta_{1}$ and $\theta_{2}$ on the contact friction coefficient between base and flowing particles, $\mu^{b}$. Please note the labels on the $x$-axis: the plot is not log-linear.

\subsection{The steady flow regime}

The $h_{\text {stop }}$ simulations yield a family of demarcation curves between arrested and steady states, $h_{\text {stop }}\left(\theta ; \mu^{b}\right)$, which can all be fitted to the Pouliquen $h_{\text {stop }}$ curve $(2)$. The fits to these curves are shown in fig. 5 ; the fitting parameters $\theta_{1}\left(\mu^{b}\right), \theta_{2}\left(\mu^{b}\right)$ and $A\left(\mu^{b}\right)$ can be found in fig. 6 . The value of $\theta_{1}$ shows little sensitivity to $\mu^{b}$, which is to be expected as $\theta_{1}$ is strongly related to the angle of repose of the material [30], which is not a function of the base configuration. For, $0 \leq \mu^{b} \leq 1 / 4, \theta_{2}$ increases as $\mu^{b}$ is increased; whereas $A$ is approximately constant, resulting in arrested flows for larger inclination angles, $\theta$, as is clearly illustrated in fig. 5 . The sensitivity of the fitting was measured by perturbing the height value of all the data points randomly by $10 \%$ and recalculating the fit. This was done 100 times and the standard deviation is used in fig. 6 to indicate the error. This $10 \%$ perturbation changed $\theta_{1}$ on average by $1.9 \%$, $\theta_{2}$ by $4.9 \%$ and $A$ by $29.5 \%$, showing that the parameter
$A$ is very sensitive to small changes in the data. As $\theta_{1}$ is related to the angle of repose, it is expect that its value should be robust to small perturbation in the data. The sensitivity in $\theta_{2}$ arises from it being an extrapolated, not interpolated, angle; therefore, small changes to the data, especially for large $\mu_{b}$, where the curve $h_{\text {stop }}(\theta)$ is very shallow for high inclinations, can cause large changes in its value. The dimensionless scale $A$ does not have a direct effect on the macroscopic friction $\mu$, it affects $\mu$ in combination with $\beta$ and $\gamma$, see (11). Small changes in the data have a large effect on both $A$ and $\gamma$, but only a small combined effect on the macroscopic friction $\mu$. This suggests that $A$ and $\gamma$ are not good parameters to characterise the friction and a better set of parameters with more physical meaning could be found. See the discussion in sect. 4.4 for more details.

\subsection{A general friction law}

In order to obtain a function for the GSLE bed macrofriction, we used the approach of Pouliquen [24] who found that for rough bases the Froude number is a linear function of $h / h_{\text {stop }}(\theta)$. Our first approach was to fit the Froude number to $h / h_{\text {stop }}\left(\theta ; \mu^{b}\right)$; however, it was found that a better collapse is obtained if the Froude number is fitted with the $h_{\text {stop }}$ curve for the case where the flowing and base particles are identical, i.e., $\mu^{f}=\mu^{b}$ such that

$$
\begin{aligned}
F=\beta\left(\mu^{b}\right) \frac{h}{h_{\text {stop }}\left(\theta ; \mu^{f}\right)}-\gamma\left(\mu^{b}\right), \\
\theta_{\text {stop }}\left(h ; \mu^{b}\right) \leq \theta \leq \theta_{a c c}\left(\mu^{b}\right),
\end{aligned}
$$

for all steady flows. In other words, when plotting $h / h_{\text {stop }}$ versus the Froude number, $F, h_{\text {stop }}\left(\theta ; \mu^{f}\right)$ was used instead of $h_{\text {stop }}\left(\theta ; \mu^{b}\right)$ because it gives a better collapse and is defined for all inclinations for which a steady flow exists. The proportionality constant, $\beta$, and offset, $\gamma$, for the fit to (10) are shown in fig. 7. The gradient $\beta$ appears to be almost independent of $\mu^{b}$; however $\gamma$ has a weak dependence slowly increasing with $\mu^{b}$. Thus, the friction coefficient of the depth-averaged eqs. (1) is given by

$$
\begin{aligned}
\mu\left(h, F ; \mu^{b}\right)= & \tan \left(\hat{\theta}_{1}\right)+\frac{\tan \left(\hat{\theta}_{2}\right)-\tan \left(\hat{\theta}_{1}\right)}{\frac{\beta\left(\mu^{b}\right) h}{\hat{A} d\left(F+\gamma\left(\mu^{b}\right)\right)}+1}, \\
& \theta_{\text {stop }}\left(h ; \mu^{b}\right) \leq \tan ^{-1} \mu \leq \theta_{\text {acc }}\left(\mu^{b}\right),
\end{aligned}
$$

where the "hat" denotes dependence on $\mu^{f}$ only, e.g., $\hat{\theta}_{1}=\theta_{1}\left(\mu^{f}\right)$, etc. The values obtained for the parameters are given in figs. 6 and 7 . The key results are that the only dependence of the macro-friction, $\mu$, on the bed contact friction, $\mu^{b}$, is through the coefficient $\gamma$, i.e., eq. (11) is valid for all steady flows, for beds with varying basal micro-friction, and only the offset $\gamma$ is a function of $\mu^{b}$, all other parameters are determined by $\mu^{f}$. A detailed investigation of how $A, \theta_{1}$ and $\theta_{2}$ depend on other parameters has been undertaken in [13]. 


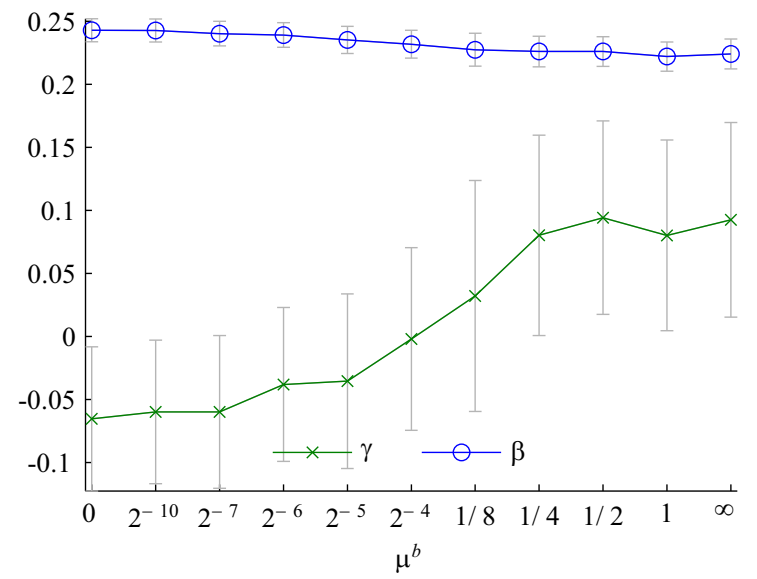

Fig. 7. Dependence of $\beta$ and $\gamma$ obtained via a fit to (10) on the contact friction coefficient between base and flowing particles $\mu^{b}$. Please note the labels on the $x$-axis: the plot is not loglinear. Error bars show $95 \%$ confidence intervals.

The values of $\beta$ and $\gamma$ reported here differ from the values reported in [13] for the case $\mu^{b}=\mu^{f}=1 / 2$. The source of this discrepancy lies in the higher resolution $h_{\text {stop }}$ curve produced here and the sensitivity of $A$ and $\theta_{2}$. The higher resolution datasets changed the $h_{\text {stop }}$ fit; however, this has the opposite effect on the values obtained for $\beta$ and $\gamma$ resulting in virtually no change in the macroscopic friction coefficient, $\mu$. This and the sensitivity of $A$ and $\gamma$ implies that $A, \theta_{2}, \beta$ and $\gamma$ may not be the best way to characterise the macroscopic friction, $\mu$.

\section{Conclusions}

An extensive parameter study of steady uniform flows was undertaken by varying height $h$, inclination $\theta$ and the basal contact friction $\mu^{b}$. At small inclinations, the flow quickly retards and a static pile is formed; at large inclinations, the flow continued to accelerate; between these two regimes there was a range of inclinations at which steady flows were observed, see fig. 1. Depth profiles for density, velocity and stress were measured using coarsegrained macroscopic fields $[12,13]$. The assumptions of depth-averaged theory are found to be valid for steady uniform flow: the density is almost constant in depth, and the downward normal and shear stress balances the gravitational forces acting on the flow (both local and in depthaveraged form).

A closure relation for the macroscopic basal friction in a shallow-granular flow model, over a geometrically rough bed, was obtained using DPM simulations, as a function of the basal and flowing particle properties. This law can be used in shallow continuum models with spatially and temporally evolving surface and mean velocity properties. Many geophysical and industrial problems involve situations where the basal roughness is not uniform and in the future it will be possible to use the presented macrofriction closure relation to perform large-scale computations (e.g., [32]) of granular flows using GSLE.
The effect of the contact law in the DPM was investigated: Hertzian, hysteretic plastic and linear springdashpot were all considered. Changing the contact law did change the fit; however, even for the nonlinear Hertzian contact model the Pouliquen flow rule remained valid (as long as some dissipation is present). The trend was, the lower the dissipation inherent in the contact model the higher $\theta_{2}$ and $\beta$, and the lower $A$.

The results of the DPM simulations did not vary significantly with the contact friction at the bed. For small values of $\mu^{b}<1 / 4$ the demarcation curves $h_{\text {stop }}\left(\theta ; \mu^{b}\right)$, $\theta_{\text {acc }}\left(\mu^{b}\right)$ between arrested, steady and accelerating flows shifted to the left, see fig. 5, implying a lower macrofriction coefficient, $\mu$. It should be noted that the fits do slightly cross near $\theta_{1}$; however, this is a fitting error and the raw data do not intersect. For $\mu^{b}<1 / 2$, the flow developed a small slip velocity at the base, see fig. 4 .

The bed friction, $\mu=\tan \theta$, was expressed as a function of height and flow velocity, cf. eq. (11). This was done using the approach of Pouliquen [24] for varying contact friction at the bed. It was found that the fits for $A$ and $\gamma$, and to a lesser degree $\beta$ and $\theta_{2}$, are very sensitive to details of the datasets and by extending the range of flows simulated the values change. However, in the friction law, eq. (4), the changes in each parameter almost cancel, resulting in only a minor change in the predicted macrofriction coefficient, $\mu$. This suggests that these parameters are not the best way to parameterise the basal friction and a better set of variables with different physical interpretation may exist.

The friction law developed here is strictly only valid for steady flows of mono-dispersed particles for the established inclination range $\theta_{\text {stop }}\left(h ; \mu^{b}\right) \leq \tan ^{-1} \mu \leq \theta_{\text {acc }}\left(\mu^{b}\right)$. However, it is anticipated that it will still hold for slightly poly-dispersed particles, slowly varying basal properties, and across a wider range of angles. The exact range of applicability of the closure law still has to be determined and this will form the theme of future work.

Both the results presented here and in [13], where the geometric basal roughness (size of basal particles) was changed, show that the flow rule for the case where bed and flow particles are the same gives the best linear collapse of Froude number against $h_{\text {stop }}$. Therefore, for the macroscopic friction coefficient, $\mu$, we propose a new hypothesis: the only dependence of $\mu$ on the base properties is through the relationship of the Froude number against $h_{\text {stop }}\left(\theta ; \mu^{f}\right)$, i.e., $\beta$ and $\gamma$. In other words, the macroscopic friction coefficient, $\mu$, is mainly determined by the properties of the flowing (bulk) material, which is surprising, since $\mu$ is a macroscopic property of the interface/wall. On the other hand, maybe the clear dependence of $\mu$ on the bulk-properties is due to the shallow-layer approach for which a height average over the bulk is intrinsic? Hence, the Pouliquen law (3) may still give insight for flows over smooth surfaces, where at the moment it is thought to be of limited applicability. This has implications for constitutive modelling and suggests a new set of experiments where different basal materials are considered. 
The authors would like to thank the late Institute of Mechanics, Processes and Control, Twente (IMPACT) for the primary financial support of this work as part of the research program "Superdispersed multiphase flows". The DPM simulations performed for this paper are undertaken in Mercury-DPM, which was initially developed within this IMPACT program. It is primarily developed by T. Weinhart, A.R. Thornton and D. Krijsman as a joint project between the Multi-Scale Mechanics (Mechanical Engineering) and the Mathematics of Computational Science (Applied Mathematics) groups at the University of Twente. We also thank the NWO VICI grant 10828 and the DFG project SPP1482 B12 for financial support. The research presented will benefit our project "Polydispersed Granular Flows through Inclined Channels" funded by STW.

Open Access This is an open access article distributed under the terms of the Creative Commons Attribution License (http://creativecommons.org/licenses/by/3.0), which permits unrestricted use, distribution, and reproduction in any medium, provided the original work is properly cited.

\section{References}

1. P.A. Cundall, O.D.L. Strack, Geotechnique 29, 47 (1979).

2. S. Luding, Granular Matter 10, 235 (2008).

3. O. Bokhove, A.R. Thornton, Handbook of Environmental Fluid Dynamics, edited by H.J. Fernando (CRC Press, 2012).

4. J.M.N.T. Gray, Y.C. Tai, S. Noelle, J. Fluid Mech. 491, 161 (2003).

5. S.B. Savage, K. Hutter, J. Fluid Mech. 199, 177 (1989).

6. C. Goujon, N. Thomas, B. Dalloz-Dubrujeaud, Eur. Phys. J. E 11, 147 (2003).

7. M.Y. Louge, S.C. Keast, Phys. Fluids 13, 1213 (2001).

8. M.Y. Louge, Phys. Rev. E 67, 061303 (2003).

9. L.E. Silbert, D. Ertas, G.S. Grest, D. Halsey, T.C. Levine, S.J. Plimpton, Phys. Rev. E. 64, 051302 (2001).
10. L.E. Silbert, J.W. Landry, G.S. Grest, Phys. Fluids 15, 1 (2003).

11. V. Kumaran, S. Maheshwari, submitted to Phys. Rev. Lett.

12. T. Weinhart, A. Thornton, S. Luding, O. Bokhove, Granular Matter 14, 289 (2012).

13. T. Weinhart, A.R. Thornton, S. Luding, O. Bokhove, Granular Matter 14, 531 (2012).

14. X. Cui, J.M.N.T. Gray, T. Johannesson, J. Geophys. Res. 112, F04012 (2007)

15. K. Dalbey, A.K. Patra, E.B. Pitman, M.I. Bursik, M.F. Sheridan, J. Geophys. Res. 113, B05203 (2008).

16. R.P. Denlinger, R.M. Iverson, J. Geophys. Res. 106, 533 (2001).

17. E.E. Doyle, A.J. Hogg, H.M. Mader, R.S.J. Sparks, Geophys. Res. Lett. 35, L04305 (2008).

18. R. Williams, A.J. Stinton, M.F. Sheridan, J. Volcan. Geotherm. Res. 177, 760 (2008).

19. J.M.N.T. Gray, X. Cui, J. Fluid Mech. 579, 113 (2007).

20. K.M. Hákonardóttir, A.J. Hogg, Phys. Fluids 17, 077101 (2005).

21. A.W. Vreman, M. Al-Tarazi, A.M. Kuipers, M. Van Sint Annaland, O. Bokhove, J. Fluid Mech. 578, 233 (2007).

22. O. Hungr, N.R. Morgenstern, Geotechnique 34, 415 (1984).

23. GDR MiDi, Eur. Phys. J. E 14, 341 (2004).

24. O. Pouliquen, Phys. Fluids 11, 542 (1999).

25. O. Pouliquen, Y. Forterre, J. Fluid Mech. 453, 131 (2002).

26. M.P. Allen, D.J. Tildesley (Editors), Computer Simulation of Liquids (Oxford University Press, 1993).

27. O.R. Walton, R.L. Braun, J. Rheol. 30, 949 (1986).

28. M. Babic, Int. J. Eng. Science 35, 523 (1997).

29. I. Goldhirsch, Granular Matter 12, 239 (2010).

30. T. Borzsonyi, R.E. Ecke, Phys. Rev. E 76, 10 (2007).

31. R.A. Bagnold, Proc. R. Soc. London, Ser. A 255, 49 (1954).

32. L. Pesch, A. Bell, W.H. Sollie, V.R. Ambati, O. Bokhove, J.J.W. Van der Vegt, ACM Trans. Math. Software 33, 4 (2007). 